\title{
Relationship between apoptotic markers in semen from fertile men and demographic, hormonal and seminal characteristics
}

\author{
Ina O Specht ${ }^{1}$, Marcello Spanò ${ }^{2}$, Karin S Hougaard ${ }^{3}$, Gian C Manicardi ${ }^{4}$, Davide Bizzaro ${ }^{5}$, Gunnar Toft ${ }^{6}$, \\ Aleksander Giwercman ${ }^{7}$ and Jens-Peter E Bonde ${ }^{1}$
}

Apoptosis in the testis has two putative roles during normal spermatogenesis; limitation of the germ cell population to numbers that can be supported by the Sertoli cells, and, possibly, selective depletion of meiotic and postmeiotic abnormal germ cells. We investigated the demographic and biological correlates of the pro-apoptotic marker Fas and the anti-apoptotic marker Bcl-xL in sperm cells of fertile men. Six hundred and four men from Greenland, Poland and Ukraine were consecutively enrolled during their pregnant wife's antenatal visits. Semen analysis was performed as recommended by the World Health Organization. Immunofluorescence coupled to flow cytometry was utilized for detection of apoptotic markers in the sperm cell. DNA damage was assessed by flow cytometry using both the sperm chromatin structure assay (SCSA) and the terminal deoxynucleotidyl transferase dUTP nick end labelling (TUNEL) assay. The percentage of Fas-positive sperm cells was higher in men with high total sperm count $(P<0.01)$, more motile sperms $(P=0.04)$ and fewer sperm head defects $(P=0.05)$. These associations were consistent within and across study regions. Furthermore, testosterone, follicle-stimulating hormone (FSH) and sexual hormone-binding globulin (SHBG) were significantly negatively correlated with Fas within and across regions as well. The data indicated no association between the anti-apoptotic Bcl-xL marker and semen or personal characteristics. The finding of Fas-positive sperm cells associated with better semen quality in a cohort of spouses of pregnant women seems different from previous data obtained in infertile men and warrants further investigation to clarify the biological significance of sperm apoptotic markers.

Asian Journal of Andrology (2012) 14, 890-896; doi:10.1038/aja.2012.76; published online 15 October 2012

Keywords: apoptosis; Bcl-x protein; Fas-associated death domain protein; fertility; sperm chromatin structure assay (SCSA); spermatozoa; TUNEL assay

\section{INTRODUCTION}

Apoptosis is the underlying mechanism of programmed death of germ cells during human spermatogenesis and is crucial for testis homeostasis during the whole reproductive lifetime. Like other cell types, male germ cells respond to external and internal stimuli by intracellular signalling pathways that ultimately determine their fate. ${ }^{1}$ Proteins of the Bcl-2 family provide one signalling pathway with some members promoting and other members antagonizing cell death. ${ }^{1,2}$ Fas/FasL provides another pathway for germ cell apoptosis. ${ }^{3}$ Apoptotic pathways converge at the level of caspases, which are considered to be the key enzymes of apoptosis, acting both as initiators and executers of programmed cell death, ${ }^{1,3}$ but caspase independent pathways have also been described. ${ }^{4}$ The finely-tuned apoptotic mechanisms eliminate abnormal cells, and thereby minimize the negative consequences of abnormal spermatozoa on male fertility and on the health of offspring.

In human semen samples, a variable fraction of sperm cells is present with DNA strand breaks. Several theories have been put forward to explain the existence of these abnormal cells, ${ }^{5-7}$ present also in otherwise normal ejaculates as assessed by the World Health Organisation's (WHO) guidelines for conventional semen analysis (WHO, 1999). It has been proposed that sperm DNA damage can originate from oxidative stress, from unligated strand breaks physiologically formed during spermatogenesis to help the process of chromatin remodelling, and from defective or 'abortive' apoptotic processes ${ }^{8-10}$ initiated post meiotically when cells are no longer able to complete the classical pathways of programmed cell death. ${ }^{1,3,11}$ Notably, it has been stressed that these mechanisms are not mutually exclusive and, in reality, DNA damage may arise from combinations of all three mechanisms. ${ }^{5}$

The theoretical framework of abortive apoptosis is based on the assumption that as germ cells progress into their terminal differentiation pathway, they become more and more transcriptionally and translationally silent and progressively lose their capacity to undergo programmed cell death in the form of classical apoptosis. Instead, a restricted form of apoptosis takes over, i.e., a sort of regulated cell

${ }^{1}$ Department of Occupational and Environmental Medicine, University Hospital of Copenhagen, Copenhagen NV DK-2400 , Denmark; ${ }^{2}$ Laboratory of Toxicology, Unit of Radiation Biology and Human Health, ENEA Casaccia Research Center, Rome I-00123, Italy; ${ }^{3}$ National Research Centre for the Working Environment, Copenhagen $\varnothing$ DK-2100, Denmark; ${ }^{4}$ Dipartimento di Scienze Agrarie e degli Alimenti, Università di Modena e Reggio Emilia, Reggio Emilia I-42121, Italy; ${ }^{5}$ Department of Life and Environmental Sciences, Polytechnic University of Marche, Ancona 1-60131, Italy and ${ }^{6}$ Department of Occupational Medicine, Aarhus University Hospital, Aarhus C DK-8000, Denmark and ${ }^{7}$ Reproductive Medicine Centre, Malmö University Hospital, Lund University, Malmö SE-20502, Sweden

Correspondence: IO Specht, (ispe0002@bbh.regionh.dk)

Received: 9 February 2012; Revised: 8 May 2012; Accepted: 22 June 2012; Published online: 15 October 2012 
death, ${ }^{5}$ mirrored by the presence of nuclear DNA strand breaks in human ejaculated spermatozoa (as assessed by terminal deoxynucleotidyl transferase dUTP nick end labelling (TUNEL) assay) and abnormal persistence of some proteins activated during the extrinsic and intrinsic apoptotic pathways, ${ }^{8,12}$ such as the pro-apoptotic marker Fas and the anti-apoptotic marker Bcl-xL. In addition, other apoptotic hallmarks, such as caspase activation and phosphatidylserine exteriorization, have been detected in human spermatozoa. ${ }^{13}$ Intriguingly, from studies where a variety of apoptotic-like sperm biomarkers were measured and correlated, it emerged that TUNEL-positive spermatozoa (which are spermatozoa with DNA breaks highlighted by labelling 3 '-OH termini) and Fas, Bcl- $\mathrm{x}$ and $\mathrm{p} 53$ expression did not always exist in unison, but semen samples that had a low sperm concentration and poor morphology were more likely to show high levels of TUNEL positivity and expression of Fas and p53. ${ }^{14}$ Even though the occurrence of this complex pattern of apoptotic-like earmarks in human sperm is far from being completely understood, the expression of sperm apoptotic markers has mainly been studied in men with infertility problems in comparison with a variety of control subjects. Limited knowledge is available from fertile population-based studies and no demographic factors influencing sperm cell apoptosis have been analysed. In this connection, applications in the field of molecular epidemiology were attempted under the hypothesis that exposures to environmental stressors could exacerbate the abnormal expression of these proteins in ejaculated human sperm. ${ }^{15-17}$

The aim of the present study was to examine demographic, hormonal and seminal correlates of the expression of the pro-apoptotic marker Fas and the anti-apoptotic marker Bcl-xL in sperm from spouses of pregnant women. We choose to analyse Fas and Bcl-xL rather than other seminal markers as caspase-3, caspase-8, caspase- 9 and phosphatidylserine externalization, because the former have been associated with male infertility in a few earlier studies of infertility clients. $^{7,8}$ A large-scale population-based study of correlates of Fas and $\mathrm{Bcl}-\mathrm{xL}$ has never been performed.

\section{MATERIALS AND METHODS}

For this cross-sectional semen study we collected semen and blood samples between May 2002 and February 2004 from 604 men with pregnant spouses in the three study regions: Greenland, Poland and Ukraine as described previously. ${ }^{18}$

\section{Study population}

In the original study, altogether 3833 pregnant women were encouraged to participate consecutively at their first antenatal care visit at three locations: (i) local hospitals in 19 cities and settlements throughout Greenland; (ii) a large central hospital in Warsaw, Poland; and (iii) three hospitals and eight antenatal clinics in Kharkiv, Ukraine.

In all countries, it was required that both partners were 18 years or more of age and born in the country of the study. The participation rate among all women who were invited to participate was $26 \%(640 / 2478)$ in Kharkiv, 68\% (472/690) in Warsaw and 90\% (598/665) in Greenland. ${ }^{15}$ In total 1710 pregnant women (45\%) were included in the study.

The male partners in this semen study were enrolled until a total of approximately 200 men had been reached in each region. ${ }^{19}$ The male partners were interviewed regarding lifestyle, occupational and reproductive history. The interview included questions regarding occupational factors, urogenital disorders, sexual abstinence and issues regarding delivery of a semen sample. Information about smoking habits, diet (seafood intake, caffeinated drinks and alcohol consumption) was obtained as well, but with reference to the period when the couple tried to become pregnant. Further details are described by Toft et al. ${ }^{18}$

The local ethical committees representing all participating populations approved the study and each subject signed an informed consent.

\section{Collection of semen and blood samples}

Participants were instructed to collect a semen sample by masturbation at their residence or in privacy in a room at the hospital after at least 2 days of sexual abstinence. Semen was collected in a sterile plastic container provide by the project team. The actual period of sexual abstinence was recorded. The sample was kept close to the body to maintain a temperature close to $37^{\circ} \mathrm{C}$ when transported to the laboratory immediately after collection. After liquefaction, and no longer than $1 \mathrm{~h}$ after ejaculation, semen samples were analyzed for concentration of spermatozoa, according to recommendations by the $\mathrm{WHO}^{19}$ and motility was analyzed according to computer-assisted sperm analysis in laboratories in the three study regions. The morphology of the sperm from the three study regions was determined centrally by two technicians at the Fertility Centre, Malmö University Hospital, on Papanicolaou-stained smears using the WHO (1999) criteria. Aliquots of the assessment of DNA damage, the expression of apoptotic markers and reproductive hormones were immediately frozen and stored at $-80^{\circ} \mathrm{C}$.

The blood samples were centrifuged immediately after collection and sera were stored at $-80^{\circ} \mathrm{C}$ for later analysis. ${ }^{19}$

\section{Determination of reproductive hormones}

Serum concentrations of follicle-stimulating hormone (FSH), luteinizing hormone (LH) and estradiol were analyzed with immunofluorometric techniques. The total assay variation coefficients were $2.9 \%, 2.6 \%$ and $8.1 \%$, respectively. Serum testosterone and sexual hormone-binding globulin (SHBG) were measured by commercially available immunoassays. The total assay variation coefficients were $5.5 \%$ and $4.6 \%$, respectively. Inhibin B levels were assessed using a specific immunometric method, as previously described, with a detection limit of $15 \mathrm{ng} \mathrm{l}^{-1}$ and intra-assay and total assay variation coefficients $<7 \%$. $^{20,21}$

All assays were performed at Malmö University Hospital (Sweden) after completion of sample collection.

\section{Determination of sperm DNA integrity}

Sperm chromatin structure assay (SCSA). Coded frozen semen samples from the three study regions were shipped on dry ice to the flow cytometry (FCM) facility of the Section of Toxicology and Biomedical Sciences (ENEA Casaccia, Rome, Italy) for SCSA analysis carried out following strictly the standardized procedure described by Evenson et al. ${ }^{22}$ Briefly, SCSA is a FCM technique that identifies the spermatozoa with abnormal chromatin packaging and/or with DNA breaks by an increased susceptibility to acid induced DNA denaturation in situ. Acridine orange (Molecular Probes, Eugene, OR, USA) staining is used to distinguish between denaturated (red fluorescence, singlestranded) and native (green fluorescence, double-stranded) DNA regions in sperm chromatin. Cells were analysed by a FACScan flow cytometer (Becton Dickinson, San José, CA, USA) and measurements were stopped when a total of 10000 events had been accumulated for each sample.

The percentage of sperm with detectable DNA fragmentation, the DNA Fragmentation Index (\%DFI), was calculated, using a dedicated software (SCSASoft; SCSA Diagnostics, Brookings, SD, USA) from the DFI frequency histogram obtained from the ratio between the red and 
total (red plus green) fluorescence intensity. ${ }^{22}$ Flow cytometer set-up and calibration were standardized using a reference semen sample as described by Spano et al. ${ }^{19}$ Interday SCSA variability, evaluated on the reference sample after 216 FCM sessions by the coefficient of variation (CV) of the \%DFI, was $6.0 \%$. In addition, 358 randomly chosen samples $(50.6 \%$ of the total) were measured twice in independent FCM sessions. Results from the two measurements were highly correlated (DFI, $r=0.96$ ).

Finally, the reliability and stability of SCSA measurements have also been challenged by an external quality control study. The quality control study was based on the blind analysis of three aliquots from seven different donors not selected among the participants in this study. The median intersample variability for \%DFI, expressed as $\mathrm{CV}$, was $1.5 \% .^{19}$

TUNEL assay. Coded frozen semen samples from the three study regions were shipped on dry ice to the FCM facility of Polytechnic University of Marche (Ancona, Italy) for TUNEL analysis.

Briefly, TUNEL assay is a method for detecting DNA fragmentation exploiting terminal deoxynucleotidyl transferase, an enzyme that catalyzes the addition of labelled dUTPs at the DNA break sites.

The pellet obtained previously was fixed in $100 \mu \mathrm{l}$ of fresh $1 \%(\mathrm{w} / \mathrm{v})$ paraformaldehyde (PFA) in PBS on a shaker for $1 \mathrm{~h}$ at $4^{\circ} \mathrm{C}$ and then washed with $4 \mathrm{ml}$ washing solution, centrifuged $\left(4^{\circ} \mathrm{C}, 700 \mathrm{~g}\right.$ for $\left.8 \mathrm{~min}\right)$ and the supernatant was discarded. The pellet was then permeabilized with $100 \mu \mathrm{l}$ of $0.1 \%(\mathrm{w} / \mathrm{v}$ ) Na-citrate/Triton-X 100 (permeabilization solution) for $2 \mathrm{~min}$ at $4^{\circ} \mathrm{C}$; subsequently, $50 \mu$ suspension was drawn for the negative control and the two new aliquots were washed with $0.1 \%(\mathrm{w} / \mathrm{v}) \mathrm{PBS} / \mathrm{BSA}$, centrifuged as above and the supernatant discarded. To both tubes, we added $40 \mu$ reaction mix (with and without the deoxynucleotidyl transferase enzyme for the positive and negative samples, respectively) and incubated for $1 \mathrm{~h}$ at $37^{\circ} \mathrm{C}$ in the dark. The pellet obtained after the washing step was fixed with $100 \mathrm{ml}$ of $0.5 \%$ (w/v) PFA containing $1 \mu \mathrm{ml}^{-1}$ propidium iodide solution in PBS. Samples were stored at $4{ }^{\circ} \mathrm{C}$ in the dark overnight until the $\mathrm{FCM}$ analyses. A minimum of 10000 sperm were measured by FCM (Epics XL flow cytometer; Beckman Coulter-IL, Fullerton, CA, USA). The intralaboratory CV regarding the TUNEL assay performance was constantly under $5 \%$. Further details are described by Stronati et al. ${ }^{17}$

\section{Determination of sperm Fas and Bcl-xL positivity}

As for the TUNEL assay, coded frozen semen samples from the three study regions were shipped on dry ice to the FCM facility of Polytechnic University of Marche (Ancona, Italy) for Fas and Bcl-xL analysis.

Sperm cells were fixed with $100 \mu \mathrm{l} 2 \%$ (w/v) PFA on a shaker for $20 \mathrm{~min}$ on ice for Bcl-xL and $1 \%$ solution for $10 \mathrm{~min}$ at $4{ }^{\circ} \mathrm{C}$ for Fas. Subsequently, for the negative control, $50 \mu \mathrm{l}$ of the suspension was drawn; the cells were washed and centrifuged as above.

To the Bcl-xL sample tube, $100 \mu$ permeabilization solution and $0.5 \%(\mathrm{w} / \mathrm{v}) \mathrm{BSA}$ with and without primary monoclonal antibody anti$\mathrm{Bcl}-\mathrm{xL}\left(20 \mu \mathrm{g} \mathrm{ml}^{-1}\right)$ were added for the sample and the negative control, respectively.

To the Fas sample tube, $40 \mu \mathrm{l}$ anti-Fas primary monoclonal antibody diluted in $0.5 \%$ (w/v) PBS/BSA and $40 \mu \mathrm{l}$ of $0.5 \%$ (w/v) PBS/BSA to the negative control tube were added.

After 1 -h incubation at $37^{\circ} \mathrm{C}$, both the samples and the negative controls were washed and the pellets resuspended in the diluted secondary antibody, for Bcl-xL $50 \mu$ l goat anti-mouse IgG-PE conjugated
$1: 100$ in PBS $+0.3 \%(w / v)$ BSA, and for Fas $100 \mu$ goat anti-mouse IgG-FITC conjugated; $1: 100$ in PBS $+0.5 \%(w / v)$ BSA and incubated for $1 \mathrm{~h}$ at $37^{\circ} \mathrm{C}$.

After the final washing step, the cells were fixed in $100 \mu \mathrm{l}$ of $0.5 \%$ PFA (w/v). The Bcl-xL samples were stained with 7-aminoactinomicyn $\mathrm{D}\left(100 \mu \mathrm{g} \mathrm{ml}^{-1}\right.$ on use) and kept in the dark at $4^{\circ} \mathrm{C}$ until run on the FCM machine $(24 \mathrm{~h})$. The Fas fixed samples were stored in the dark at $4^{\circ} \mathrm{C}(24 \mathrm{~h})$ and $15 \mathrm{~min}$ before the FCM analysis, the suspension was stained with $1 \mu \mathrm{g} \mathrm{ml}^{-1}$ propidium iodide.

Two different control ejaculates, stored frozen at $-80^{\circ} \mathrm{C}$ in the laboratory, were thawed and processed according to the $\mathrm{Bcl}-\mathrm{xL}$ and Fas protocol before starting every FCM run which ensured standardization and stability of the instruments. The intralaboratory $\mathrm{CV}$ regarding the apoptotic markers was in the range from $6 \%$ for Fas to $9 \%$ for Bcl-xL. ${ }^{17}$

All reagents used were molecular biology grade. The primary and secondary antibodies were from Instrumentation Laboratory (Milan, Italy); BSA fraction V, PBS, Na-citrate, Triton X-100, propidium iodide from Sigma Pharmaceuticals (Sigma-Aldrich, Milan, Italy), 7AAD from Molecular Probes (Invitrogen, Milan, Italy) and secondary antibody blocking peptide from Santa Cruz Biotechnology (CA, USA).

The amount of missing values for Bcl-xL and Fas from Poland (49.7\% and $37.6 \%$, respectively) and Ukraine $(78.4 \%$ and $32.7 \%$, respectively) were high due to accidental loss of samples during shipment and insufficient number of cells for analysis. In Greenland, $27.6 \%$ and $3.0 \%$ of Bcl-xL and Fas were missing due to few cells for analysis as well.

A minimum of 10000 sperm were measured by FCM (Epics XL flow cytometer; Beckman Coulter-IL).

\section{Statistical analysis}

General linear models (Proc GLM) were used to analyze crude and adjusted associations of demographic, seminal and hormonal characteristics with the two apoptotic markers, Fas and Bcl-xL. Analysis of trends between levels of determinants and apoptotic markers were based on continuous variables.

Observations from the three study regions, Greenland, Poland and Ukraine, were initially analysed separately but are presented in aggregate because correlations were similar across regions as observed by scatter plots. Results for the separate analysis in each study region will not be shown.

Demographical characteristics included study region, age groups $(\leqslant 25,25-34, \geqslant 34$ years), body mass index $(\leqslant 24.9,24.9-29.9$, $\geqslant 29.9 \mathrm{~kg} \mathrm{~m}^{-2}$ ), caffeinated drinks ( $\leqslant 5,>5 \mathrm{cups} /$ day), sexual abstinence time ( $\leqslant 3,>3$ days), spillage during semen collection (yes/no), cotinine in serum $\left(\leqslant 1.4,1.4-190, \geqslant 190 \mathrm{ng} \mathrm{ml}^{-1}\right)$, fever during the last three month (yes/no), genital infections (yes/no) and testicular disorders (yes/no). Seminal characteristics included DFI $(\leqslant 10,10-20$, $\geqslant 20 \%)$, TUNEL $(0-3,3-10,>10 \%)$, total sperm count $(\leqslant 60,60$ $\left.120, \geqslant 120 \times 10^{6}\right)$, sperm concentration $(\leqslant 20, \quad 20-40, \geqslant 40 \times$ $\left.10^{6} \mathrm{ml}^{-1}\right)$, volume $(\leqslant 2,2-4, \geqslant 4 \mathrm{~g})$, immature sperm cells $(\leqslant 1$, $>1 \%)$, sperm cells with normal morphology $(\leqslant 4,4-14, \geqslant 14 \%)$, head defects $(\leqslant 90,90-95, \geqslant 95 \%)$, midpiece defects $(\leqslant 10$, $10-20, \geqslant 20 \%)$, tail defects $(\leqslant 5,5-10, \geqslant 10 \%)$, motility $(\leqslant 50$, $>50 \%)$, Fas $(\leqslant 10,10-50, \geqslant 50 \%)$ and Bcl-xL $(\leqslant 5,5-40, \geqslant 40 \%)$. Seminal covariates were categorized according to clinical cut-off values or tertiles. All hormonal characteristics were divided into tertiles and included testosterone, estradiol, inhibin-B, FSH, LH and SHBG. 
The two outcome variables, Fas and Bcl-xL, were analyzed on a continuous scale. Residuals of Fas and Bcl-xL were checked for normality and were transformed by the natural logarithm.

All crude analyses were adjusted for study region. In the multiple general linear models, we adjusted for study region, age groups, body mass index, caffeinated drinks, cotinine in serum, fever during the last three month, sexual abstinence time, genital infections, testicular disorders and spillage during semen sampling, all divided as described above.

Statistical analysis was performed with SAS software, version 9.1 for Windows (SAS Institute Inc., Cary, NC, USA).

\section{RESULTS}

Four hundred and fifty-four men from Greenland, Poland and Ukraine had completed Fas results $(3.0 \%, 37.6 \%$ and $32.7 \%$ missing, respectively), whereas $\mathrm{Bcl}-\mathrm{xL}$ observations only were available for 243 men from Greenland and Poland $(27.6 \%$ and $49.7 \%$ missing, respectively).

Men from Poland had approximately a doubling of percentage Faspositive sperm cells compared with Greenland and Ukraine. Otherwise no demographic characteristics were associated with percentage of Fas-positive sperm cells (Table 1). However, several seminal characteristics and reproductive hormones were associated with Fas-positive sperm cells in both bivariate analysis and after adjustment for demographic and clinical characteristics (Tables 2 and 3). Sperm concentration, total sperm count $(P=0.01)$ and motility $(P=0.04)$ were positive correlated with Fas, whereas sperm head defects were negatively correlated with Fas $(P=0.05)$, both before and after adjustment for potential confounding effects (Table 2). Normal sperm morphology was in the crude analysis significant positively correlated with Fas $(P=0.04)$, but not after adjustment $(P=0.07)$ (Table 2). Results were consistent across study regions except the correlation between sperm count and Fas, which was weaker in Poland and Ukraine (data not shown). Fas positivity was not associated with sperm DNA damage either evaluated by SCSA or the TUNEL assay.

Negative correlations between FSH, testosterone, SHBG and Fas were statistically significant $(P<0.05)$ with correlation coefficients near -0.2 (Table 3 ). The three correlations were all consistent within each study region (data not shown). Only FSH was statistically significant when grouped into tertiles $(P=0.021)$ (Table 3 ).

$\mathrm{Bcl}-\mathrm{xL}$ and Fas were positively correlated on a continuous scale $(r=0.2, P<0.05)$ (data not shown). We found no significant associations between any reproductive hormones, demographical and/or clinical characteristics and the anti-apoptotic marker Bcl-xL (data not shown).

\section{DISCUSSION}

This study is to our knowledge the first large study of seminal apoptotic markers in a population of fertile men. Our results show that better semen quality is correlated with a higher percentage of Faspositive spermatozoa.

Previous studies have investigated Fas expression of ejaculated spermatozoa among infertile men or in men presenting different degrees of alterations in semen parameters compared with fertile controls. In the majority of the studies, Fas was not found on ejaculated sperm cells in normozoospermic men, ${ }^{23-25}$ or if present, only in low percentages. ${ }^{26}$ Some studies did not even observe Fas on spermatozoa from infertile men ${ }^{23}$ or only at low percentages. ${ }^{25}$ Studies have found Fas-positive sperm cells in infertile men, and reported inverse relationships between Fas and normal sperm cell morphology, concentration and
Table 1 Association between Fas and demographic and clinical characteristics

\begin{tabular}{|c|c|c|c|c|c|c|}
\hline & & Fas $\%$ & positivity & & & \\
\hline & $n$ & Mean & Median & Range $^{a}$ & $\mathrm{P}_{c r d}$ & $\mathrm{P}_{a d j}^{b}$ \\
\hline Site & & & & & & \\
\hline Greenland & 193 & 25.0 & 19.0 & 91 & $<0.01$ & $<0.01$ \\
\hline Poland & 123 & 48.6 & 42.3 & 98 & & \\
\hline Ukraine & 138 & 28.2 & 17.3 & 98 & & \\
\hline Age (year) & & & & & & \\
\hline $18-25$ & 117 & 27.0 & 17.9 & 95 & 0.49 & 0.67 \\
\hline $26-34$ & 239 & 34.3 & 24.3 & 98 & & \\
\hline $35-51$ & 92 & 35.2 & 23.6 & 96 & & \\
\hline Body mass in & & & & & & \\
\hline $18.5-24.9$ & 221 & 29.1 & 18.9 & 98 & 0.10 & 0.12 \\
\hline 25-29.9 & 180 & 34.4 & 24.6 & 98 & & \\
\hline$>30$ & 48 & 39.6 & 33.3 & 95 & & \\
\hline Caffeinated dr & & & & & & \\
\hline$<5$ & 284 & 33.2 & 22.8 & 98 & 0.95 & 0.70 \\
\hline$>5$ & 146 & 30.7 & 21.2 & 98 & & \\
\hline Alcohol (drink & & & & & & \\
\hline$<14$ & 239 & 37.3 & 26.6 & 98 & 0.11 & 0.07 \\
\hline$>14$ & 32 & 27.1 & 17.8 & 80 & & \\
\hline Current smok & & & & & & \\
\hline Yes & 265 & 29.5 & 19.0 & 98 & 0.31 & 0.24 \\
\hline No & 185 & 36.4 & 26.8 & 98 & & \\
\hline Cotinine (ng $n$ & & & & & & \\
\hline$<1.4$ & 185 & 35.9 & 24.0 & 98 & 0.60 & 0.73 \\
\hline $1.5-190.0$ & 121 & 32.9 & 24.9 & 98 & & \\
\hline$>190$ & 148 & 27.6 & 18.0 & 98 & & \\
\hline Fever & & & & & & \\
\hline Yes & 45 & 31.4 & 26.6 & 97 & 0.50 & 0.30 \\
\hline No & 400 & 32.6 & 22.2 & 98 & & \\
\hline Genital infecti & & & & & & \\
\hline Yes & 175 & 25.0 & 19.0 & 94 & 0.05 & 0.20 \\
\hline No & 279 & 37.0 & 24.3 & 98 & & \\
\hline Testicular disc & & & & & & \\
\hline Yes & 10 & 28.3 & 17.6 & 94 & 0.55 & 0.95 \\
\hline No & 444 & 32.5 & 22.9 & 98 & & \\
\hline
\end{tabular}

a The range is the difference between the highest and lowest number of Fas in each category of demographic or clinical characteristics.

${ }^{\mathrm{b}}$ Adjusted for site, age groups, body mass index, caffeinated drinks, cotinine in serum, fever the last three month, spillage during semen collection, sexual abstinence time, genital infections and testicular disorders.

${ }^{c}$ Genital infections: epididymitis, gonorrhea, chlamydia or mumps in adulthood.

${ }^{\mathrm{d}}$ Testicular disorders: treatment for retracted testis, surgery for varicose veins, torsio testis or testis cancer.

motility. ${ }^{14,24,26}$ These findings indicate that abortive apoptosis may have taken place. Thus, our results are not concordant with previous results. In fertile men from the present study, Fas was present in $96 \%$ of the semen samples and correlated with semen parameters, indicating that high Fas levels were positively associated with good semen quality. This could indicate that spermatozoa of fertile men are resistant to Fas-mediated apoptosis and that Fas is inactive or expresses molecules that inhibit the signal induced by Fas or the death program itself. ${ }^{27,28}$ In fact, Fas-mediated apoptosis is partially inhibited by overexpression of Bcl-2. ${ }^{27}$ However, this does not explain the positive associations observed in the present study. Our observations, however, support a study of three fertile bulls, where high levels of Fas antigen in ejaculated spermatozoa were found. This suggests that Fas antigen in mature, normal ejaculated spermatozoa might evoke a non-apoptotic response against cell death stimuli or to cell-damaging micro-environmental 
Table 2 Association between Fas and seminal characteristics

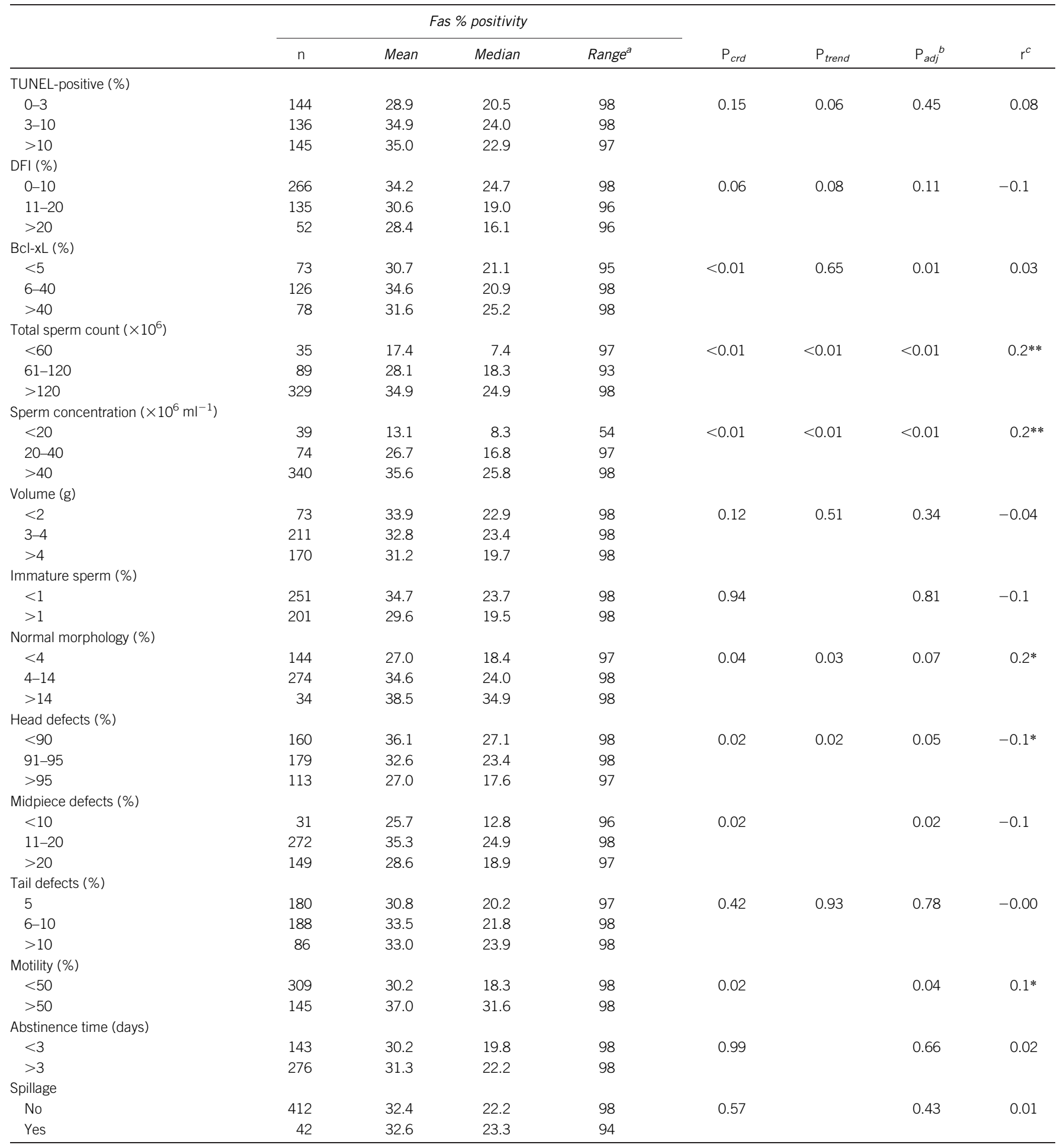

Abbreviations: DFI, DNA Fragmentation Index; TUNEL, terminal deoxynucleotidyl transferase dUTP nick end labelling.

${ }^{a}$ The range is the difference between the highest and lowest number of Fas in each category of seminal characteristics.

${ }^{\mathrm{b}}$ Adjusted for site, age groups, body mass index, caffeinated drinks, cotinine in serum, fever the last three month, spillage during semen collection, sexual abstinence time, genital infections and testicular disorders.

${ }^{c}$ Spearmans rank correlation $(* P<0.05, * * P<0.0001)$.

factors like oxidative stress. ${ }^{29}$ The same authors also found FasL (the Fas ligand) on ejaculated spermatozoa, indicating co-expression of Fas and FasL in the same cells. ${ }^{30}$ They hypothesized that Fas and FasL play different, independent roles in sperm cells. Possible FasL in bull spermatozoa is able to kill activated lymphocytes, and thereby protect the male gamete from damage by the immune system or by the cytotoxic 
Table 3 Association between Fas and reproductive hormones

\begin{tabular}{|c|c|c|c|c|c|c|c|c|c|}
\hline \multicolumn{10}{|c|}{ Fas $\%$ positively } \\
\hline & $\mathrm{n}$ & Mean & Median & Range $^{a}$ & $\mathrm{P}_{c r d}$ & $\mathrm{P}_{\text {trend }}$ & $\mathrm{P}_{a d j}^{b}$ & $\mathrm{P}_{\text {trend }}$ & $r^{c}$ \\
\hline \multicolumn{10}{|c|}{ Testosterone (nmol I-1) } \\
\hline $13-18$ & 124 & 33 & 23 & 98 & & & & & \\
\hline$>18$ & 111 & 24 & 16 & 94 & & & & & \\
\hline \multicolumn{10}{|c|}{ Estradiol (pmol $\mathrm{I}^{-1}$ ) } \\
\hline$>81$ & 110 & 33 & 23 & 96 & & & & & \\
\hline \multicolumn{10}{|c|}{ Inhibin-B (ng $\left.\right|^{-1}$ ) } \\
\hline $22-150$ & 114 & 33 & 21 & 98 & 0.09 & 0.43 & 0.051 & 0.10 & 0.02 \\
\hline 150-203 & 129 & 33 & 23 & 98 & & & & & \\
\hline$>203$ & 114 & 31 & 23 & 97 & & & & & \\
\hline \multicolumn{10}{|l|}{$\mathrm{FSH}\left(\mathrm{IU} \mathrm{I}^{-1}\right)$} \\
\hline $3-5$ & 113 & 33 & 24 & 98 & & & & & \\
\hline$>5$ & 120 & 30 & 20 & 98 & & & & & \\
\hline \multicolumn{10}{|c|}{ SHBG (nmol I $\left.{ }^{-1}\right)$} \\
\hline $6-21$ & 121 & 38 & 27 & 98 & 0.07 & 0.004 & 0.28 & 0.07 & $-0.21 *$ \\
\hline $21-31$ & 117 & 34 & 24 & 98 & & & & & \\
\hline$>31$ & 118 & 26 & 18 & 98 & & & & & \\
\hline
\end{tabular}

Abbreviations: FSH, follicle-stimulating hormone; LH, luteinizing hormone; SHBG, sexual hormone-binding globulin.

${ }^{a}$ The range is the difference between the highest and lowest number of Fas in each category of reproductive hormones.

${ }^{b}$ Adjusted for site, age groups, body mass index, caffeinated drinks, cotinine in serum, fever the last three month, spillage during semen collection, sexual abstinence time, genital infections and testicular disorders.

${ }^{\mathrm{c}}$ Spearmans rank correlation $(* P<0.05)$.

activity of leukocytes in the female genital tract. ${ }^{30}$ It would be of great interest to investigate if FasL is present on human ejaculated spermatozoa from fertile men.

In the present study, we did not find associations or determinants of $\mathrm{Bcl}-\mathrm{xL}$ and demographic, seminal characteristics or reproductive hormones. Likewise, previous studies investigating pro-apoptotic proteins in the $\mathrm{Bcl}-2$ family did not observe associations between $\mathrm{Bcl}$ and sperm concentration, morphology or motility. ${ }^{12}$ However, Fas and Bcl-xL were positively correlated. Our observations support findings by Sakkas et al. ${ }^{14}$ with significant positive correlations between Fas and Bcl-x. Bcl-x can be either the long (xL) or the short (xS) form. They investigated which form was expressed in their samples. Bcl-x and $\mathrm{Bcl}-\mathrm{xL}$ were significantly correlated indicating that $\mathrm{Bcl}-\mathrm{xL}$ was present. Concomitant high levels of $\mathrm{Bcl}$ and Fas might indicate that $\mathrm{Bcl}$ is activated in an attempt to turn off apoptosis.

Like in our study, Sakkas et al. ${ }^{14}$ did not find an association between Fas and TUNEL positivity. Our results also lacked association with the SCSA, the other assay deployed to assess independently sperm chromatin integrity. Furthermore, other studies have not found any relationship between Fas and DNA damage. ${ }^{24}$ In a study of 620 Chinese men seeking fertility treatment the authors hypothesized that genetic variants in Fas, FasL and Casp8 might contribute to abnormal spermatogenic apoptosis, measured by TUNEL assay, followed by poor semen quality, which could give rise to male infertility. ${ }^{31}$ They found that polymorphisms of Fas-670GG and Casp8-652 6N ins/del were associated with low level of apoptosis in sperm cells and poor semen quality. Germ cells from men carrying both the Fas-670G and Casp8$6526 \mathrm{~N}$ del alleles, i.e., with reduced expression of both Fas and Casp8, displayed lower levels of apoptosis compared to those carrying only one of the alleles. ${ }^{31}$ These men are therefore at the lowest risk of sperm apoptosis. ${ }^{31}$ A study of Fas, FasL polymorphisms and azoospermia or severe oligoospermia found that individuals with FasL-844TT genotype were more susceptible to azoospermia or severe oligozoospermia than those with FasL-844CC genotype. ${ }^{32}$ These findings indicate that apoptosis of germ cells may be dependent on genetic diversity. It would therefore be relevant to compare genotypes in fertile and infertile men.

Cross-sectional studies of semen quality suffer often of low participation rates which may bias the internal validity of a study. Subfertile men might be more motivated to participate, but the nature of this cross-sectional design, with data collection through two years, might be predominated by highly fertile couples. However, selection bias among participating and non-participating men is unlikely, since the length of time to pregnancy did not differ among couples where men provided a semen sample in comparison with couples where men declined to deliver semen. ${ }^{17}$

The participation rate of women in the original study varied from $90 \%$ in Greenland to $26 \%$ in Ukraine. The low participation rate in Kharkiv was a consequence of the recruitment procedure where contact between potential participants and the project team were managed by approximately 30 medical doctors at the three hospitals and eight antenatal clinics. With this large organization, a high level of information and encouragement to participate was not possible. Demographic and reproductive information was obtained from a sample of 605 of those women that declined participation in the study. Only the average age was slightly lower among non-participating women $[(22.8 \pm 2.4)$ versus $(24.9 \pm 2.8)$ years $] .{ }^{15}$ 
The amount of missing Fas and Bcl-xL from especially Poland and Ukraine were high due to accidental loss of samples during shipment and insufficient numbers of cells for analysis. The loss was random and is thus not expected to bias the results.

The contrasting results from this and previous studies, i.e., high levels of Fas in men with good semen quality and the high percentage of Fas-positive spermatozoa in the ejaculate, might be due to different study populations and/or possible methodological differences in cell fixation, primary antibody clone, fluorescence-labeled secondary antibodies, FCM analysis, software for the off-line analysis to obtain the fraction of positive cells, and the criteria used to define positivity from the FCM data.

This might also indicate that there are differences in the molecular markers of apoptosis between males with normal and abnormal semen parameters and that Fas receptor molecules might be non-functional or non-operative after ejaculation or even protecting the spermatozoa.

\section{CONCLUSION}

Ejaculated spermatozoa exhibiting DNA damage do not necessarily show distinct apoptotic markers. Apoptosis and DNA damage in fertile and subfertile men may therefore not be strictly related. These results, carried out in a cohort of spouses of pregnant women, indicate that Fas-positive sperm cells are associated with better semen quality. The results seem different from previous data obtained in subfertile men and warrant further investigation to clarify the biological significance of sperm apoptotic markers.

\section{AUTHOR CONTRIBUTIONS}

The study was conceived by GCM, DB, GT and JPB. All authors contributed to design and analyses. Laboratory analyses of apoptotic markers and TUNEL assay were performed by DB and GCM, and MS performed analyses of DNA fragmentation index. GT and AG were responsible for conventional semen analysis and AG was responsible for lab analyses of hormones. IOS, KSH and JPB compiled the data and performed biostatisical analyses. IOS drafted a first version of manuscript and tables and all authors contributed with amendments and revisions.

\section{COMPETING FINANCIAL INTERESTS}

None of the authors have competing financial interest in the results of this study.

\section{ACKNOWLEDGMENTS}

The project was supported by grants from European Commission to the 5th and 7th Framework Programs (INUENDO, contract No. QLK4-CT-200100202 and CLEAR, FP7-ENV-2008-1 contract No. 226217), the Danish Research Council (No.10-082745) and the Reprosund project.

1 Tripathi R, Mishra DP, Shaha C. Male germ cell development: turning on the apoptotic pathways. J Reprod Immunol 2009; 83: 31-5.

2 Print CG, Loveland KL. Germ cell suicide: new insights into apoptosis during spermatogenesis. Bioessay 2000; 22: 423-30.

3 Shaha C, Tripathi R, Mishra DP. Male germ cell apoptosis: regulation and biology. Philos Trans R Soc Lond B Biol Sci 2010; 365: 1501-15.

4 Taylor SL, Weng SL, Fox P, Duran EH, Morshedi MS et al. Somatic cell apoptosis markers and pathways in human ejaculated sperm: potential utility as indicators of sperm quality. Mol Hum Reprod 2004; 10: 825-34.
5 Aitken RJ, De luliis GN. On the possible origins of DNA damage in human spermatozoa. Mol Hum Reprod 2010; 16: 3-13.

6 Barratt CL, Aitken RJ, Bjorndahl L, Carrell DT, de Boer P et al. Sperm DNA: organization, protection and vulnerability: from basic science to clinical applications-a position report. Hum Reprod 2010; 25: 824-38.

7 Sakkas D, Alvarez JG. Sperm DNA fragmentation: mechanisms of origin, impact on reproductive outcome, and analysis. Fertil Steril 2010; 93: 1027-36.

8 Sakkas D, Mariethoz E, Manicardi G, Bizzaro D, Bianchi PG et al. Origin of DNA damage in ejaculated human spermatozoa. Rev Reprod 1999; 4: 31-7.

9 Sakkas D, Seli E, Bizzaro D, Tarozzi N, Manicardi GC. Abnormal spermatozoa in the ejaculate: abortive apoptosis and faulty nuclear remodelling during spermatogenesis. Reprod Biomed Online 2003; 7: 428-32.

10 Sakkas D, Seli E, Manicardi GC, Nijs M et al. The presence of abnormal spermatozoa in the ejaculate: did apoptosis fail? Hum Fertil (Camb) 2004; 7: 99-103

11 Aitken RJ, Koppers AJ. Apoptosis and DNA damage in human spermatozoa. Asian J Androl 2011; 13: 36-42.

12 Cayli S, Sakkas D, Vigue L, Demir R, Huszar G. Cellular maturity and apoptosis in human sperm: creatine kinase, caspase- 3 and $\mathrm{Bcl}-\mathrm{XL}$ levels in mature and diminished maturity sperm. Mol Hum Reprod 2004; 10: 365-72.

13 Weng SL, Taylor SL, Morshedi M, Schuffner A, Duran EH et al. Caspase activity and apoptotic markers in ejaculated human sperm. Mol Hum Reprod 2002; 8: 984-91.

14 Sakkas D, Moffatt O, Manicardi GC, Mariethoz E, Tarozzi N et al. Nature of DNA damage in ejaculated human spermatozoa and the possible involvement of apoptosis. Biol Reprod 2002; 66: 1061-7.

15 Bonde JP, Toft G, Rylander L, Rignell-Hydbom A, Giwercman A et al. Fertility and markers of male reproductive function in Inuit and European populations spanning large contrasts in blood levels of persistent organochlorines. Environ Health Perspect 2008; 116: 269-77.

16 Long M, Stronati A, Bizzaro D, Kruger T, Manicardi GC et al. Relation between serum xenobiotic-induced receptor activities and sperm DNA damage and sperm apoptotic markers in European and Inuit populations. Reproduction 2007; 133: 517-30.

17 Stronati A, Manicardi GC, Cecati M, Bordicchia M, Ferrante L et al. Relationships between sperm DNA fragmentation, sperm apoptotic markers and serum levels of CB153 and p,p'-DDE in European and Inuit populations. Reproduction 2006; 132: 949-58.

18 Toft G, Axmon A, Giwercman A, Thulstrup AM, Rignell-Hydbom A et al. Fertility in four regions spanning large contrasts in serum levels of widespread persistent organochlorines: a cross-sectional study. Environ Health 2005; 4: 26.

19 Spano M, Toft G, Hagmar L, Eleuteri P, Rescia M et al. Exposure to PCB and p, p'-DDE in European and Inuit populations: impact on human sperm chromatin integrity. Hum Reprod 2005; 20: 3488-99.

20 Groome NP, Illingworth PJ, O'Brien M, Pai R, Rodger FE et al. Measurement of dimeric inhibin B throughout the human menstrual cycle. J Clin Endocrinol Metab 1996; 81: 1401-5.

21 Rignell-Hydbom A, Rylander L, Giwercman A, Jonsson BA, Nilsson-Ehle $P$ et al. Exposure to CB-153 and p, $p^{\prime}-\mathrm{DDE}$ and male reproductive function. Hum Reprod 2004; 19: 2066-75.

22 Evenson DP, Larson KL, Jost LK. Sperm chromatin structure assay: its clinical use for detecting sperm DNA fragmentation in male infertility and comparisons with other techniques. J Androl 2002; 23: 25-43.

23 Castro A, Parodi D, Morales I, Madariaga M, Rios R et al. Absence of Fas protein detection by flow cytometry in human spermatozoa. Fertil Steril 2004; 81: 1019-25.

24 McVicar CM, McClure N, Williamson K, Dalzell LH, Lewis SE. Incidence of Fas positivity and deoxyribonucleic acid double-stranded breaks in human ejaculated sperm. Fertil Steril 2004; 81: 767-74.

25 Perticarari S, Ricci G, Boscolo R, De SM, Pagnini G et al. Fas receptor is not present on ejaculated human sperm. Hum Reprod 2008; 23: 1271-9.

26 Sakkas D, Mariethoz E, St John JC. Abnormal sperm parameters in humans are indicative of an abortive apoptotic mechanism linked to the Fas-mediated pathway. Exp Cell Res 1999; 251: 350-5.

27 Nagata S, Golstein P. The Fas death factor. Science 1995; 267: 1449-56.

28 Wajant H, Pfizenmaier K, Scheurich P. Non-apoptotic Fas signaling. Cytokine Growth Factor Rev 2003; 14: 53-66.

29 Meggiolaro D, Porcelli F, Carnevali A, Crepaldi P, Savarese E et al. A possible role of Fas antigen in ejaculated spermatozoa of fertile bulls: an immunocytochemical quantitative approach. Acta Histochem 2006; 107: 463-8.

30 Porcelli F, Meggiolaro D, Carnevali A, Ferrandi B. Fas ligand in bull ejaculated spermatozoa: a quantitative immunocytochemical study. Acta Histochem2006; 108: 287-92.

$31 \mathrm{Ji} \mathrm{G}, \mathrm{Gu}$ A, Hu F, Wang S, Liang J et al. Polymorphisms in cell death pathway genes are associated with altered sperm apoptosis and poor semen quality. Hum Reprod 2009; 24: 2439-46.

32 Wang W, Lu N, Xia Y, Gu A, Wu B et al. FAS and FASLG polymorphisms and susceptibility to idiopathic azoospermia or severe oligozoospermia. Reprod Biomed Online2009; 18: 141-7. 